\title{
Boundedness and stability of discrete Volterra equations
}

\author{
Małgorzata Migda ${ }^{1 *}$, Miroslava Růžičková $^{2}$ and Ewa Schmeidel ${ }^{3}$
}

\footnotetext{
"Correspondence:

malgorzata.migda@put.poznan.pl

1 Poznan University of Technology, Poznan, Poland

Full list of author information is available at the end of the article
}

\section{Abstract}

In this paper, using the fixed point theorem, we investigate the boundedness and asymptotic stability of the zero solution of the discrete Volterra equation

$$
x(n+1)=a(n)+b(n) x(n)+\sum_{i=n_{0}}^{n} K(n, i) x(i)
$$

Necessary conditions for the existence of a periodic solution of the above equation are also obtained.

MSC: 39A10; 39A22; 39A30

Keywords: Volterra difference equation; stability; bounded solution; periodic solution; contraction mapping

\section{Introduction}

Let $\mathbb{N}, \mathbb{Z}, \mathbb{R}$ denote the set of nonnegative integers, the set of all integers and the set of real numbers, respectively, and $\mathbb{N}_{0}=\left\{n_{0}, n_{0}+1, \ldots\right\}$, where $n_{0} \in \mathbb{N}$.

In this paper we consider the linear discrete Volterra equation of non-convolution type of the form

$$
x(n+1)=a(n)+b(n) x(n)+\sum_{i=n_{0}}^{n} K(n, i) x(i), \quad n \geq n_{0},
$$

where $a, b: \mathbb{N}_{0} \rightarrow \mathbb{R}, b(n) \neq 0$ for $n \geq n_{0}, K: \mathbb{N}_{0} \times \mathbb{N}_{0} \rightarrow \mathbb{R}$. By a solution of equation (1) we mean a sequence $x: \mathbb{N}_{0} \rightarrow \mathbb{R}$ satisfying equation (1) for all $n \geq n_{0}$.

We introduce some notation and definitions that will be used in the paper. Hereafter, we denote the solution of equation (1) with the initial condition $x\left(n_{0}\right)=x_{0}$ by $x\left(n, n_{0}, x_{0}\right)$.

Definition 1 Solutions of equation (1) are equi-bounded if for each constant $M_{1}>0$, there is $M_{2}>0$ such that $\left|x_{0}\right| \leq M_{1}$ and $n \geq n_{0}$ implies that $\left|x\left(n, n_{0}, x_{0}\right)\right| \leq M_{2}$.

If $a(n) \equiv 0$ in equation (1), then (1) takes the following form:

$$
x(n+1)=b(n) x(n)+\sum_{i=n_{0}}^{n} K(n, i) x(i), \quad n \geq n_{0} .
$$

\section{Springer}

(C) 2015 Migda et al.; licensee Springer. This is an Open Access article distributed under the terms of the Creative Commons Attribution License (http://creativecommons.org/licenses/by/4.0), which permits unrestricted use, distribution, and reproduction in any medium, provided the original work is properly credited. 
Definition 2 The zero solution of equation (2) is stable if for every $\varepsilon>0$ there exists $\delta>0$ such that $\left|x_{0}\right| \leq \delta$ implies $\left|x\left(n, n_{0}, x_{0}\right)\right| \leq \varepsilon$ for $n \geq n_{0}$.

Definition 3 The zero solution of equation (2) is asymptotically stable if there exists $\mu>0$ such that $\left|x_{0}\right| \leq \mu$ implies $\lim _{n \rightarrow \infty} x(n)=0$.

Denote the Banach space of all bounded real sequences $x: \mathbb{N}_{0} \rightarrow \mathbb{R}$ equipped with the supremum norm $\|x\|=\sup _{n \geq n_{0}}|x(n)|$ by $B S$.

We will also adopt the customary notations

$$
\sum_{i=k+s}^{k} u(i)=0, \quad \prod_{i=k+s}^{k} u(i)=1,
$$

where $k$ is an integer, $s$ is a positive integer and $u$ is a sequence of real numbers.

Volterra difference equations are widely used in modeling of processes in many branches of natural sciences, economics and engineering. Some important properties of their solutions are boundedness, stability and periodicity. Boundedness of solutions of linear discrete Volterra equations has been studied by many authors. For example, Kolmanovskii and Shaikhet [1] have established a necessary and sufficient condition for boundedness of all solutions of the linear Volterra equation

$$
\Delta x(n)=\sum_{i=0}^{n} A(n, i) x(i), \quad n \geq 0,
$$

where $A(j, i) \geq 0, j \geq i \geq 0$.

Appleby et al. [2] found sufficient conditions in terms of the kernel $H$ under which every solution of the system of linear Volterra equations

$$
x(n+1)=h(n)+\sum_{i=0}^{n} H(n, i) x(i), \quad n \geq 0,
$$

is convergent. The necessary and sufficient conditions for boundedness of all solutions of the above equation are given by Győri and Reynolds in [3].

In [4], Khandaker and Raffoul studied the stability properties of the zero solution of the nonlinear Volterra discrete system

$$
x(n+1)=A(n) x(n)+\sum_{s=0}^{n} B(n, s) x(s)+g(n, x(n))
$$

by expressing the solution in terms of the resolvent matrix. Here, the authors assumed that $|g(n, x(n))| \leq \lambda(n)|x(n)|$, where $\lambda(n)$ is such that $0 \leq \lambda(n) \leq N<\infty$ for some constant $N$. A scalar case of the above equation is also considered in Section 4 of Raffoul [5].

Yankson, in [6], studied the asymptotic stability of the zero solution of the Volterra difference delay equation

$$
x(n+1)=a(n) x(n)+c(n) \Delta x(n-g(n))+\sum_{s=n-g(n)}^{n-1} k(n, s) h(x(s)) .
$$


Since the condition $0 \leq g(n) \leq g_{0}$ is assumed, then equation (1) cannot be obtained as a special case of this equation.

Boundedness of solutions of linear and nonlinear discrete Volterra equations was also studied by Crisci et al. [7], Diblík and Schmeidel [8], Gronek and Schmeidel [9], Győri and Awwad [10], Győri and Horváth [11], Kolmanovskii and Shaikhet [1], Medina [12], Migda and Migda [13] or Migda and Morchało [14]. A survey of the fundamental results on the stability of linear Volterra difference equations, of both convolution and non-convolution type, can be found in Elaydi [15], see also Crisci et al. [16] and [17]. The problem of finding periodic and asymptotically periodic solutions of linear discrete Volterra equations of type (1) was investigated, for example, by Diblik et al. [8, 18-21], Elaydi [22], Gajda et al. [23], Győri and Reynolds [24], Migda and Migda [13] or Song and Baker [25].

Motivated by the results obtained in the papers by Islam and Yankson [26] and Raffoul [27], in this paper, we derive explicit sufficient conditions for the equi-boundedness of solutions of equation (1) and the asymptotic stability of the zero solution of equation (2).

We prove our main results using the variation of constants formula and the contraction mapping principle. We study necessary conditions for the existence of periodic solutions. Our results generalize certain results obtained in [9] and [1]. Moreover, they can be applied to equations for which the results obtained in some of the above mentioned papers could not be used (see Examples 1-3).

\section{Boundedness and stability}

Let us note that by the variation of constants formula, the solution of equation (1) satisfies the following equation:

$$
\begin{aligned}
x(n)= & x_{0} \prod_{j=n_{0}}^{n-1} b(j)+\sum_{k=n_{0}}^{n-1}\left(\prod_{j=k+1}^{n-1} b(j)\right) \sum_{i=n_{0}}^{k} K(k, i) x(i) \\
& +\sum_{k=n_{0}}^{n-1}\left(\prod_{j=k+1}^{n-1} b(j)\right) a(k) \quad \text { for } n \geq n_{0},
\end{aligned}
$$

where $x\left(n_{0}\right)=x_{0}$. Using this fact we prove the following theorems.

Theorem 1 Suppose that

$$
\sum_{n=n_{0}}^{\infty}|a(n)|<\infty
$$

and there exists a nonnegative real constant B such that

$$
\prod_{i=n_{0}}^{n-1}|b(i)| \leq B, \quad n \geq n_{0} .
$$

Assume also that there exists $\alpha \in(0,1)$ such that

$$
\sum_{k=n_{0}}^{n-1} \prod_{j=k+1}^{n-1}|b(j)| \sum_{i=n_{0}}^{k}|K(k, i)| \leq \alpha \quad \text { for } n \geq n_{0} .
$$

Then the solutions of equation (1) are equi-bounded. 
Proof By (4), there exists a nonnegative real constant $A$ such that $\sum_{n=n_{0}}^{\infty}|a(n)|=A$. Let $M_{1}>0$ be given. Choose a real number $M_{2}$ such that $\frac{\left(A+M_{1}\right) B}{1-\alpha} \leq M_{2}$. Let $u_{0}$ be a real number such that $\left|u_{0}\right| \leq M_{1}$. We define a subset $S$ of $B S$ by

$$
S=\left\{x \in B S: x_{0}=u_{0} \text { and }\|x\| \leq M_{2}\right\} .
$$

Then $S$ is a closed subset of $B S$. Now, we define the mapping $T: S \rightarrow B S$ as follows:

$$
\begin{aligned}
(T x)(n)= & u_{0} \prod_{j=n_{0}}^{n-1} b(j)+\sum_{k=n_{0}}^{n-1}\left(\prod_{j=k+1}^{n-1} b(j)\right) \sum_{i=n_{0}}^{k} K(k, i) x(i) \\
& +\sum_{k=n_{0}}^{n-1}\left(\prod_{j=k+1}^{n-1} b(j)\right) a(k) \quad \text { for } n \geq n_{0} .
\end{aligned}
$$

We will prove that $T$ has a fixed point in $S$.

Firstly, we show that $T(S) \subset S$. Indeed, if $x \in S$, then by (7), (6), (4) and (5), we get

$$
\begin{aligned}
|(T x)(n)| \leq & M_{1} \prod_{j=n_{0}}^{n-1}|b(j)| \\
& \quad+M_{2} \sum_{k=n_{0}}^{n-1} \prod_{j=k+1}^{n-1}|b(j)| \sum_{i=n_{0}}^{k}|K(k, i)|+\prod_{j=n_{0}}^{n-1}|b(j)| \sum_{k=n_{0}}^{n-1}|a(k)| \\
\leq & B M_{1}+\alpha M_{2}+B A \leq M_{2} \quad \text { for } n \geq n_{0} .
\end{aligned}
$$

Moreover, we have $(T x)\left(n_{0}\right)=u_{0}$. Hence, $T(x) \in S$ and $T(S) \subset S$.

Next, we prove that $T$ is a contraction. Let $y, z \in S$. Then, using (6) and (7), we get

$$
\begin{aligned}
|(T y)(n)-(T z)(n)| & \leq \sum_{k=n_{0}}^{n-1} \prod_{j=k+1}^{n-1}|b(j)| \sum_{i=n_{0}}^{k}|K(k, i)||y(i)-z(i)| \\
& \leq \sup _{n \geq n_{0}}|y(n)-z(n)|\left(\sum_{k=n_{0}}^{n-1} \prod_{j=k+1}^{n-1}|b(j)| \sum_{i=n_{0}}^{k}|K(k, i)|\right) \\
& \leq \alpha \sup _{n \geq n_{0}}|y(n)-z(n)| \quad \text { for } n \geq n_{0} .
\end{aligned}
$$

Thus

$$
\|T y-T z\|=\sup _{n \geq n_{0}}|(T y)(n)-(T z)(n)| \leq \alpha\|y-z\| .
$$

Hence, $T$ is a contraction. By the contraction mapping principle, $T$ has a unique fixed point $x^{*}$ in $S$. It means that $\left(T x^{*}\right)(n)=x^{*}(n)$. So, from (7) we have

$$
x^{*}(n)=u_{0} \prod_{j=n_{0}}^{n-1} b(j)+\sum_{k=n_{0}}^{n-1}\left(\prod_{j=k+1}^{n-1} b(j)\right) \sum_{i=n_{0}}^{k} K(k, i) x^{*}(i)+\sum_{k=n_{0}}^{n-1}\left(\prod_{j=k+1}^{n-1} b(j)\right) a(k) .
$$


By comparing the above equation and (3), we see that the unique fixed point $x^{*}$ is also a solution of equation (1). Hence, by the definition of the set $S$, the solutions of equation (1) are equi-bounded. This completes the proof.

Theorem 1 extends Theorem 2.1 in [1] and Theorem 1 in [9].

The following example illustrates the result presented in Theorem 1.

Example 1 Let us consider the linear Volterra difference equation

$$
x(n+1)=\frac{1}{n(n+1)}+x(n)+\sum_{i=1}^{n} \frac{i}{n^{2}(n+1)^{2}} x(i), \quad n \geq 1 .
$$

Here

$$
a(n)=\frac{1}{n(n+1)}, \quad b(n) \equiv 1 \quad \text { and } \quad K(n, i)=\frac{i}{n^{2}(n+1)^{2}} .
$$

Hence $A=\sum_{n=1}^{\infty} \frac{1}{n(n+1)}=1, B=\prod_{j=1}^{\infty} b(j)=1$,

$$
\sum_{k=1}^{n-1} \prod_{j=k+1}^{n-1}|b(j)| \sum_{i=1}^{k}|K(k, i)|=\sum_{k=1}^{n-1} \frac{1}{k^{2}(k+1)^{2}} \sum_{i=1}^{k} i=\frac{1}{2} \sum_{k=1}^{n-1} \frac{1}{k(k+1)} \leq \frac{1}{2}=\alpha .
$$

So, by Theorem 1 the solutions of (1) are equi-bounded. For example, for $M_{1}=1$ we have $M_{2} \geq \frac{\left(A+M_{1}\right) B}{1-\alpha}=4$. Hence, all solutions with the initial condition $|x(1)| \leq 1$ have the property $\|x\| \leq 4$.

Note that for equation (8), Theorem 1 in [28] cannot be applied since the assumption given below of this theorem

$$
|b(n)|+|K(n, n)|+\varphi(0) \leq 1-\alpha,
$$

where $\alpha \in(0,1), \varphi(n) \geq 0, n \geq 1$, is not satisfied.

In the next example we present a Volterra difference equation for which condition (24) from [2] does not hold. Hence, Theorem 5.1 [2] is not applicable for this example whereas our Theorem 1 is.

Example 2 Let us consider the linear Volterra difference equation

$$
x(n+1)=\left\lfloor\frac{n+1}{2}\right\rfloor^{(-1)^{n}} x(n)+\sum_{i=1}^{n} \frac{1}{2^{k+i}} x(i), \quad n \geq 1 .
$$

Here

$$
b(n)=\left\lfloor\frac{n+1}{2}\right\rfloor^{(-1)^{n}}=\left(1,1, \frac{1}{2}, 2, \frac{1}{3}, 3, \frac{1}{4}, 4, \ldots\right) \text { and } K(n, i)=\frac{1}{2^{n+i}} .
$$

It is easy to verify that conditions (5) and (6) are satisfied. So, by Theorem 1 the solutions of (9) are equi-bounded. 
Remark 1 From Theorem 3.1 in [7] it follows that the boundedness of equation (2) is equivalent to the stability of its zero solution. Hence, and by Theorem 1 , conditions (5) and (6) ensure the stability of the zero solution of equation (2).

Now, we provide conditions for the asymptotic stability of the zero solution of equation (2).

Theorem 2 If condition (6) holds and

$$
\lim _{n \rightarrow \infty} \prod_{i=n_{0}}^{n-1} b(i)=0
$$

then the zero solution of equation (2) is asymptotically stable.

Proof By (10) there exists a constant $B_{1}$ such that $\left|\prod_{i=n_{0}}^{n-1} b(i)\right| \leq B_{1}$ for $n \geq n_{0}$. Let $M>0$ be given. Choose $\mu>0$ such that

$$
\mu \leq \frac{(1-\alpha) M}{B_{1}}
$$

Let $u_{0}$ be a real number such that $\left|u_{0}\right| \leq \mu$. We define a subset $S_{1}$ of $B S$ by

$$
S_{1}=\left\{x \in B S: x_{0}=u_{0},\|x\| \leq M \text { and } \lim _{n \rightarrow \infty} x(n)=0\right\}
$$

Then $S_{1}$ is a closed subset of $B S$. We define the mapping $T_{1}$ for $x \in S_{1}$ as follows:

$$
\left(T_{1} x\right)(n)=u_{0} \prod_{j=n_{0}}^{n-1} b(j)+\sum_{k=n_{0}}^{n-1}\left(\prod_{j=k+1}^{n-1} b(j)\right) \sum_{i=n_{0}}^{k} K(k, i) x(i) \quad \text { for } n \geq n_{0} .
$$

We show that $T_{1}\left(S_{1}\right) \subset S_{1}$. Indeed, if $x \in S_{1}$, then by (6) and (11) we have

$$
\begin{aligned}
\left|\left(T_{1} x\right)(n)\right| & \leq \mu \prod_{j=n_{0}}^{n-1}|b(j)|+M \sum_{k=n_{0}}^{n-1} \prod_{j=k+1}^{n-1}|b(j)| \sum_{i=n_{0}}^{k}|K(k, i)| \\
& \leq \mu B_{1}+M \alpha \leq M \quad \text { for } n \geq n_{0} .
\end{aligned}
$$

Moreover, from (10) we immediately get that the first term on the right-hand side of (12) tends to zero. We show that

$$
\left|\sum_{k=n_{0}}^{n-1}\left(\prod_{j=k+1}^{n-1} b(j)\right) \sum_{i=n_{0}}^{k} K(k, i) x(i)\right| \rightarrow 0 \quad \text { as } n \rightarrow \infty .
$$

Let $\varepsilon>0$. Since $\lim _{n \rightarrow \infty} x(n)=0$, there exists $n_{1} \geq n_{0}$ such that

$$
\sup _{n \geq n_{1}}|x(n)| \leq \frac{\varepsilon}{2 \alpha}
$$


By (10) there exists $n_{2} \geq n_{1} \geq n_{0}$ such that

$$
\prod_{j=n_{2}}^{n-1}|b(j)| \leq \frac{\varepsilon}{2 \alpha M} \quad \text { for } n \geq n_{2}
$$

Then, for $n \geq n_{2}$, we have

$$
\begin{aligned}
\mid \sum_{k=n_{0}}^{n-1} & \left(\prod_{j=k+1}^{n-1} b(j)\right) \sum_{i=n_{0}}^{k} K(k, i) x(i) \mid \\
\leq & \sum_{k=n_{0}}^{n-1} \prod_{j=k+1}^{n-1}|b(j)| \sum_{i=n_{0}}^{k}|K(k, i)||x(i)| \\
\leq & \sum_{k=n_{0}} \prod_{j=k+1}^{n_{2}-1}|b(j)| \sum_{i=n_{0}}^{n-1}|K(k, i)||x(i)| \\
& +\sum_{k=n_{2}}^{n-1} \prod_{j=k+1}^{n-1}|b(j)| \sum_{i=n_{0}}^{k}|K(k, i)||x(i)| \\
\leq & \sup _{n \geq n_{0}}|x(n)| \sum_{k=n_{0}}^{n_{2}-1} \prod_{j=k+1}^{n_{2}-1}|b(j)| \prod_{j=n_{2}}^{n-1}|b(j)| \sum_{i=n_{0}}^{k}|K(k, i)| \\
& +\sup _{n \geq n_{2}}|x(n)| \sum_{k=n_{2}}^{n-1} \prod_{j=k+1}^{n-1}|b(j)| \sum_{i=n_{0}}^{k}|K(k, i)| .
\end{aligned}
$$

From the above, the definition of the set $S_{1},(6),(13)$ and (14), we obtain

$$
\left|\sum_{k=n_{0}}^{n-1}\left(\prod_{j=k+1}^{n-1} b(j)\right) \sum_{i=n_{0}}^{k} K(k, i) x(i)\right| \leq M \prod_{j=n_{2}}^{n-1}|b(j)| \alpha+\frac{\varepsilon}{2 \alpha} \alpha \leq \frac{\varepsilon}{2}+\frac{\varepsilon}{2}=\varepsilon .
$$

Thus, the second term of formula (12) tends to zero, too. So, we have $\lim _{n \rightarrow \infty}(T x)(n)=0$. Hence, $T_{1}(x) \in S_{1}$ and $T_{1}\left(S_{1}\right) \subset S_{1}$.

Similarly as in the proof of Theorem 1, we get that $T$ has a fixed point. This fixed point solves equation (1) and tends to zero. Hence, the zero solution of equation (2) is asymptotically stable.

Example 3 Let us consider the linear Volterra difference equation

$$
x(n+1)=\frac{1}{n} x(n)+\sum_{i=1}^{n} \frac{1}{2 n} x(i), \quad n \geq 1 .
$$

Here

$$
b(n)=\frac{1}{n} \quad \text { and } \quad K(n, i)=\frac{1}{2 n} .
$$

It is easy to verify that conditions (5) and (6) are satisfied. So, by Theorem 1 the solutions of (15) are equi-bounded. Moreover, since condition (10) is also satisfied, by Theorem 2 the zero solution of equation (15) is asymptotically stable. 
Note that the result about asymptotic stability of the zero solution obtained in the section Scalar Equation (see Example 3.1) of [4] is not applicable here because assumption vi) $\sum_{n=1}^{\infty}|K(n, i)| \leq C$, where $C$ is a positive constant, for (15) is not satisfied. Similarly, Theorem 2.7 of [7] could not be applied for (15) since the kernel $K(n, i)=\frac{1}{2 n}$ does not satisfy condition (2.28).

In Proposition 3.2 of [3], the necessary and sufficient conditions for boundedness of all solutions of equations of type (2) are given. It is easy to see that for (15), assumption (17) of [3] does not hold. But, as it was shown above, each solution of this equation is bounded. Corollary 3.7 of [3] is not applicable here, too.

\section{Existence of periodic solutions}

In this section we give necessary conditions for the existence of periodic solutions of equation (1) assuming the periodicity conditions

$$
a(n+q)=a(n), \quad b(n+q)=b(n), \quad n \in \mathbb{N}_{0}
$$

and

$$
K(n+q, i)=K(n, i)=K(n, i+q), \quad n, i \in \mathbb{N}_{0},
$$

for some integer $q \geq 1$. If condition (17) is satisfied, then the sequence $K$ is said to be doubly $q$-periodic sequence.

Denote $P_{q}=\{x \in B S: x(n+q)=x(n)\}$. Let us consider the system of linear equations

$$
\left\{\begin{array}{l}
a_{11} x\left(n_{0}\right)+a_{12} x\left(n_{0}+1\right)+\cdots+a_{1 q} x\left(n_{0}+q-1\right)=0 \\
a_{21} x\left(n_{0}\right)+a_{22} x\left(n_{0}+1\right)+\cdots+a_{2 q} x\left(n_{0}+q-1\right)=0 \\
\vdots \\
a_{q 1} x\left(n_{0}\right)+a_{q 2} x\left(n_{0}+1\right)+\cdots+a_{q q} x\left(n_{0}+q-1\right)=0
\end{array}\right.
$$

where

$$
a_{i j}=K\left(n_{0}+i-1, n_{0}+j-1\right) \quad \text { for } i, j \in\{1,2, \ldots, q\}
$$

and $x\left(n_{0}\right), x\left(n_{0}+1\right), \ldots, x\left(n_{0}+q-1\right)$ are the unknowns. Denote the fundamental matrix of system (18) by $A_{q}$, i.e.,

$$
A_{q}=\left(\begin{array}{cccc}
K\left(n_{0}, n_{0}\right) & K\left(n_{0}, n_{0}+1\right) & \ldots & K\left(n_{0}, n_{0}+q-1\right) \\
K\left(n_{0}+1, n_{0}\right) & K\left(n_{0}+1, n_{0}+1\right) & \ldots & K\left(n_{0}+1, n_{0}+q-1\right) \\
\vdots & \vdots & \ddots & \vdots \\
K\left(n_{0}+q-2, n_{0}\right) & K\left(n_{0}+q-2, n_{0}+1\right) & \ldots & K\left(n_{0}+q-2, n_{0}+q-1\right) \\
K\left(n_{0}+q-1, n_{0}\right) & K\left(n_{0}+q-1, n_{0}+1\right) & \ldots & K\left(n_{0}+q-1, n_{0}+q-1\right)
\end{array}\right) .
$$

In the next theorem we give necessary conditions for the existence of a periodic solution of equation (1). 
Theorem 3 Let conditions (16) and (17) hold, and let $A_{q}$ be the matrix defined by (19). If there exists a q-periodic solution of equation (1), then $\operatorname{det} A_{q}=0$.

Proof Let $x \in P_{q}$ be a nontrivial solution of equation (1). Then, by (1), we have

$$
x(n+1)=x(n+q+1)=a(n+q)+b(n+q) x(n+q)+\sum_{i=n_{0}}^{n+q} K(n+q, i) x(i) .
$$

Hence, using (16) and (17), we get

$$
\begin{aligned}
x(n+1) & =a(n)+b(n) x(n)+\sum_{i=n_{0}}^{n} K(n, i) x(i)+\sum_{i=n+1}^{n+q} K(n, i) x(i) \\
& =x(n+1)+\sum_{i=n+1}^{n+q} K(n, i) x(i) .
\end{aligned}
$$

Thus, by the $q$-periodicity of $x$ and $K$, we get

$$
\sum_{i=r+1}^{r+q} K(r, i) x(i)=0, \quad \text { for } r=n, n+1, \ldots, n+q-1 .
$$

This means that the above system has a nontrivial solution for any $n \geq n_{0}$. By the periodicity of the sequences $x$ and $K$, system (20) is equivalent to system (18). Hence, system (18) has a nontrivial solution, too. Thus $\operatorname{det} A_{q}=0$. This completes the proof.

Remark 2 From the proof of Theorem 3 it follows that if $x$ is a $q$-periodic solution of (1), then $x$ has the following form

$$
x(n)=\left(c_{1}, c_{2}, \ldots, c_{q}, c_{1}, c_{2}, \ldots, c_{q}, \ldots\right),
$$

where $\left(c_{1}, c_{2}, \ldots, c_{q}\right)$ is a certain solution of system (18).

Example 4 Let us consider the linear Volterra difference equation of the form (1), where sequences $a, b, K$ are 3-periodic, $n_{0}=1$ and

$$
\begin{aligned}
& a(3 k+1)=1, \quad a(3 k+2)=-3, \quad a(3 k+3)=2, \quad k=0,1, \ldots, \\
& b(3 k+1)=-2, \quad b(3 k+2)=2, \quad b(3 k+2)=1, \quad k=0,1, \ldots,
\end{aligned}
$$

and

$$
A_{3}=\left(\begin{array}{lll}
1 & 0 & 1 \\
2 & 2 & 2 \\
0 & 1 & 0
\end{array}\right)
$$

The considered equation has a 3-periodic solution $x$ of the form

$$
x(3 k+1)=1, \quad x(3 k+2)=0, \quad x(3 k+3)=-1 .
$$

It is easy to check that $\operatorname{det} A_{3}=0$ and the triple $(1,0,-1)$ is a solution of system (18). 
The next example shows that the condition $\operatorname{det} A_{q}=0$ is not sufficient for the existence of a $q$-periodic solution of (1).

Example 5 Let us consider the linear Volterra difference equation of the form (1), where sequences $a, b, K$ are 2-periodic, $n_{0}=1$ and

$$
\begin{aligned}
a(2 k+1) & =-1, \quad a(2 k+2)=1, \quad k=0,1, \ldots \\
b(2 k+1) & =2, \quad b(2 k+2)=1, \quad k=0,1, \ldots \\
A_{2} & =\left(\begin{array}{ll}
2 & 1 \\
4 & 2
\end{array}\right)
\end{aligned}
$$

Then $\operatorname{det} A_{2}=0$, but it is easy to check that the considered equation does not have a 2periodic solution.

For some periodic difference equations and systems, see also, for example, $[29,30]$ or [31].

\section{Competing interests}

The authors declare that they have no competing interests.

\section{Authors' contributions}

The authors declare that the study was realized in collaboration with the same responsibility. All authors read and approved the final manuscript.

\section{Author details}

${ }^{1}$ Poznan University of Technology, Poznan, Poland. ${ }^{2}$ University of Zilina, Zilina, Slovakia. ${ }^{3}$ University of Bialystok, Bialystok, Poland.

\section{Acknowledgements}

The first author was supported by the project PB-43-081/14DS of Ministry of Science and Higher Education of Poland.

Received: 16 October 2014 Accepted: 6 January 2015 Published online: 20 February 2015

\section{References}

1. Kolmanovskii, V, Shaikhet, L: Some conditions for boundedness of solutions of difference Volterra equations. Appl. Math. Lett. 16, 857-862 (2003)

2. Appleby, JAD, Győri, I, Reynolds, DW: On exact convergence rates for solutions of linear systems of Volterra difference equations. J. Differ. Equ. Appl. 12, 1257-1275 (2006)

3. Győri, I, Reynolds, DW: Sharp conditions for boundedness in linear discrete Volterra equations. J. Differ. Equ. Appl. 15, 1151-1164 (2009)

4. Khandaker, TM, Raffoul, YN: Stability properties of linear Volterra discrete systems with nonlinear perturbation. J. Differ. Equ. Appl. 8, 857-874 (2002)

5. Raffoul, YN: General theorems for stability and boundedness for nonlinear functional discrete systems. J. Math. Anal. Appl. 279(2), 639-650 (2003)

6. Yankson, E: Stability of Volterra difference delay equations. Electron. J. Qual. Theory Differ. Equ. 2006, Article ID 20 (2006)

7. Crisci, MR, Kolmanovskii, VB, Russo, E, Vecchio, A: Boundedness of discrete Volterra equation. J. Math. Anal. Appl. 211, 106-130 (1997)

8. Diblík, J, Schmeidel, E: On the existence of solutions of linear Volterra difference equations asymptotically equivalent to a given sequence. Appl. Math. Comput. 218(18), 9310-9320 (2012)

9. Gronek, T, Schmeidel, E: Existence of bounded solution of Volterra difference equations via Darbo's fixed-point theorem. J. Differ. Equ. Appl. 19(10), 1645-1653 (2013)

10. Győri, I, Awwad, E: On the boundedness of the solutions in nonlinear discrete Volterra difference equations. Adv. Differ. Equ. 2012, Article ID 2 (2012). doi:10.1186/1687-1847-2012-2

11. Győri, I, Horváth, L: Asymptotic representation of the solutions of linear Volterra difference equations. Adv. Differ. Equ. 2008, Article ID 932831 (2008)

12. Medina, R: Asymptotic behavior of Volterra difference equations. Comput. Math. Appl. 41(5-6), 679-687 (2001)

13. Migda, M, Migda, J: Bounded solutions of nonlinear discrete Volterra equations. Math. Slovaca (in press)

14. Migda, M, Morchało, J: Asymptotic properties of solutions of difference equations with several delays and Volterra summation equations. Appl. Math. Comput. 220, 365-373 (2013)

15. Elaydi, S: Stability and asymptoticity of Volterra difference equations: a progress report. J. Comput. Appl. Math. 228(2), 504-513 (2009) 
16. Crisci, MR, Jackiewicz, Z, Russo, E, Vecchio, A: Stability analysis of discrete recurrence equations of Volterra type with degenerate kernels. J. Math. Anal. Appl. 162, 49-62 (1991)

17. Crisci, MR, Kolmanovskii, VB, Russo, E, Vecchio, A: Stability of difference Volterra equations: direct Liapunov method and numerical procedure. Comput. Math. Appl. 36(10-12), 77-97 (1998)

18. Diblík, J, Rüžičková, M, Schmeidel, E: Asymptotically periodic solutions of Volterra difference equations. Tatra Mt. Math. Publ. 43, 43-46 (2009)

19. Diblík, J, Rižžičková, M, Schmeidel, E: Existence of asymptotically periodic solutions of Volterra system difference equations. J. Differ. Equ. Appl. 15(11-12), 1165-1177 (2009)

20. Diblík, J, Rủžičková, M, Schmeidel, E: Asymptotically periodic solutions of Volterra systems of difference equations. Comput. Math. Appl. 59(8), 2854-2867 (2010)

21. Diblík, J, Rǔžičková, M, Schmeidel, E, Zbąszyniak, M: Weighted asymptotically periodic solutions of linear Volterra difference equations. Abstr. Appl. Anal. 2011, Article ID 37098 (2011). doi:10.1155/2011/370982

22. Elaydi, S: Periodicity and stability of linear Volterra difference systems. J. Math. Anal. Appl. 181, 483-492 (1994)

23. Gajda, K, Gronek, T, Schmeidel, E: On the existence of a weighted asymptotically constant solutions of Volterra difference equations of nonconvolution type. Discrete Contin. Dyn. Syst., Ser. B 19(8), 2681-2690 (2014)

24. Györi, I, Reynolds, DW: On asymptotically periodic solutions of linear discrete Volterra equations. Fasc. Math. 44, 53-67 (2010)

25. Song, Y, Baker, CTH: Perturbations of Volterra difference equations. J. Differ. Equ. Appl. 10, 379-397 (2004)

26. Islam, MN, Yankson, E: Boundedness and stability in nonlinear delay difference equations employing fixed point theory. Electron. J. Qual. Theory Differ. Equ. 2005, Article ID 26 (2005)

27. Raffoul, YN: Stability and periodicity in discrete delay equations. J. Math. Anal. Appl. 324, 1356-1362 (2006)

28. Raffoul, YN: Boundedness and periodicity of Volterra systems of difference equations. J. Differ. Equ. Appl. 4(4), 381-393 (1998)

29. Diblík, J, Hlavičková, l: Asymptotic behavior of solutions of delayed difference equations. Abstr. Appl. Anal. 2011 Article ID 671967 (2011). doi:10.1155/2011/671967

30. Stevič, S, Diblík, J, Iričanin, B, Šmarda, Z: On a periodic system of difference equations. Abstr. Appl. Anal. 2012, Article ID 258718 (2012)

31. Stevič, S, Diblík, J, Šmarda, Z: On periodic and solutions converging to zero of some systems of differential-difference equations. Appl. Math. Comput. 227, 43-49 (2014)

\section{Submit your manuscript to a SpringerOpen ${ }^{\circ}$ journal and benefit from:}

- Convenient online submission

Rigorous peer review

- Immediate publication on acceptance

- Open access: articles freely available online

- High visibility within the field

- Retaining the copyright to your article 